\title{
Presence of Basic Oscillatory Properties in the Bagley-Torvik Model
}

\author{
Temirkhan Aleroev and Ludmila Kirianova*
}

Moscow State University of Civil Engineering, Yaroslavskoe shosse, 26, Moscow, 129337, Russia

\begin{abstract}
The article is devoted to the finding and analysis by numerical methods of eigenvalues and eigenfunctions of the boundary value problem of the differential equation of the second order containing the operator of fractional differentiation in the Riemann - Liouville sense, the so called Bagley - Torvik model. This model is used to analyze damping properties of various viscoelastic materials.
\end{abstract}

\section{Introduction}

Consider the differential equation of the form:

$$
u^{\prime \prime}(x)+c D^{\alpha} u(x)+\lambda u(x)=0,
$$

suppose that the boundary conditions are satisfied:

$$
u(0)=0 ; u(1)=0
$$

There $D^{\alpha} u(x)$ is the fractional differentiation operator in the Riemann-Liouville sense, the order of which $\alpha$ is assumed to belong to the interval from 1 to 2 , that is,

$$
D^{\alpha} u(x)=\frac{d^{2}}{d x^{2}}\left[\frac{1}{\Gamma(2-\alpha)} \int_{0}^{t} \frac{u(\tau) d \tau}{(x-\tau)^{\alpha-1}}\right], 1<\alpha<2 .
$$

This article is devoted to the study of eigenfunctions and eigenvalues of problem (1) (3).

The studied equation (1) is usually called the Bagley - Torvik equation, since it was first considered in 1983 by Bagley and Torvick [1,2] in studies on modeling the damping properties of viscoelastic materials, such as polymers, glasses, etc. The study of the basic oscillation properties of a physical system described by the Bagley - Torvik equation has now been devoted to a considerable number of papers, for example [3-11]. It should be noted that the results obtained in this direction refer to the case when the order of the fractional derivative is $0<\alpha<1$. In the case where $1<\alpha<2$, the basic oscillatory properties of the system described by equation (1) have not been established. Although this

\footnotetext{
* Corresponding author: ludmilakirianova@yadex.ru
} 
case simulates the change in the deformation-strength properties of polymer concrete, since the order of the fractional derivative in such a model $\alpha=1,47$.

These papers aroused great interest, which has not weakened until now [12]. On the basis of this model, predictive estimates are made, which find their application in various fields, for example, in construction for the introduction of amendments to existing rules and regulations, in geological exploration in the search for uranium-containing ores, in radioecology in the assessment of radon danger of territories and buildings, in geophysics in the study of lithospheric-atmospheric relations, the search for earthquake precursors.

In [13], the Bagley - Torvik model was used in modeling the change in deformationstrength characteristics of polymer concrete under loading. Samples of polymer concrete based on polyester resin (diane and dichlorohydride-1,1-dichloro-2,2-diethylene) were investigated. Polymer concrete is represented as a set of granules of mineral filler, which are in a viscoelastic medium. In this case, the movement of the granule is described by equation (1), where $\mathrm{c}$ is the modulus of the resin viscosity, is the modulus of the resin hardness, and $\alpha$ is the viscoelasticity parameter of the medium.

\section{Results}

First of all, we note that in the paper of the authors [14] the following initial problem for the Bagley - Torvik equation was investigated for the case $1<\alpha<2$

$$
\begin{gathered}
u^{\prime \prime}(x)+c D^{\alpha} u(x)+\lambda u(x)=0 . \\
u(0)=0, u^{\prime}(0)=1 .
\end{gathered}
$$

Using a recurrent kernels sequence, we solved the solution of problem (4) - (5) in the form of a power series

$$
u(x)=x-\sum_{n=1}^{\infty} \sum_{m=0}^{n}(-1)^{n+1} \frac{c_{n}^{m} c^{m} \lambda^{n-m} x^{2 n+1-m \alpha}}{\Gamma(2 n-m \alpha+2)} .
$$

In the same paper, an analytic formula was obtained for calculating the eigenvalues of this problem:

$$
\lambda_{k}=k^{2}-c \cdot k^{\alpha} \sin \frac{(1-\alpha) \pi}{2} .
$$

and also the corresponding eigenfunctions are written out:

$$
u_{k}(x)=u\left(x, \lambda_{k}\right)=x-\sum_{n=1}^{\infty} \sum_{m=0}^{n}(-1)^{n+1} \frac{c_{n}^{m} c^{m} \lambda_{k}^{n-m} x^{2 n+1-m \alpha}}{\Gamma(2 n-m \alpha+2)} .
$$

In the case where $1<\alpha<2$, the formulas similar to (7) - (8) cannot be obtained for eigenvalues and eigenfunctions. Therefore, for this case the authors investigated the influence function $G_{2}(x, \tau)$ of this problem, described in detail in [13]:

$$
G_{2}(x, \tau)=G_{1}(x, \tau)-\frac{c}{\mathrm{E}_{1 / \beta}(c, 2)} \int_{\tau}^{1} \mathrm{E}_{\rho}[c(\eta-\tau)]^{\beta} d \eta \cdot \int_{0}^{1} G(x, t) D_{0 t}^{\alpha-1} \mathrm{E}_{\beta}\left[c t^{\beta}\right] d t,
$$

where 


$$
\begin{gathered}
\beta=\frac{1}{2-\alpha}, \quad G(x, t)=\left\{\begin{array}{l}
t(x-1), t \leq x \\
x(t-1), t>x
\end{array}\right. \\
G_{1}(x, \tau)=\left\{\begin{array}{l}
(1-x) \int_{\tau}^{x} \mathrm{E}_{\beta}[c(t-\tau)] d t-x-\int_{x}^{1} \mathrm{E}_{\beta}[c(t-\tau)]^{\beta} d t, x \geq \tau, \\
(1-x) \int_{\tau}^{x} \mathrm{E}_{\beta}[c(t-\tau)] d t-x \int_{\tau}^{1} \mathrm{E}_{\beta}[c(t-\tau)]^{\beta} d t, \quad x<\tau,
\end{array}\right.
\end{gathered}
$$

and

$\mathrm{E}_{\rho}[z, \mu]=\sum_{m=0}^{\infty} \frac{z^{m}}{\Gamma\left(\mu+\frac{m}{\rho}\right)}-$ is a well-known function of Mittag-Leffler type,

$\mathrm{E}_{\rho}[z]=\mathrm{E}_{1 / \rho}[z, 1]-$ is a function of Mittag-Leffler.

Analysis of the influence function made it possible to show that the first eigenvalue of problem (1) - (3) is positive and simple, and the fundamental tone has no nodes. Using numerical methods, the eigenvalues and eigenfunctions of the problem describing the change in the deformation-strength properties of polymer concrete, studied in [13], are written out as follows:

1) for $\lambda_{k}$

$$
\sum_{n=1}^{52} \sum_{m=0}^{n}(-1)^{n+1} \frac{C_{n}^{m}(1,8)^{m} \lambda_{k}^{n-m}}{\Gamma(2 n-1,47 m+2)}=1
$$

2) for $u_{k}(x)$

$$
u_{k}(x)=x-\sum_{n=1}^{52} \sum_{m=0}^{n}(-1)^{n+1} \frac{C_{n}^{m}(1,8)^{m} \lambda_{k}^{n-m} x^{2 n+1-1,47 m}}{\Gamma(2 n-1,47 m+2)} .
$$

Next, using numerical methods, we find the eigenvalues (with the accuracy of one decimal place) and for $\alpha=1,47$ and $c=1,8$ the corresponding eigenfunctions of problem (1) - (2) using the high-level language of technical calculations MATLAB and build their graphs (note again that in this case the Bagley -Torvik model describes the change in the deformation-strength properties of polymer concrete).

The eigenvalues are presented in the table 1.

Table 1. The eigenvalues (with the accuracy of one decimal place) the Bagley-Torvik model for $\alpha=1,47$ and $c=1,8$.

\begin{tabular}{|c|c|}
\hline Designations & Meanings \\
\hline$\lambda_{1}$ & 16,6 \\
\hline$\lambda_{2}$ & 59,5 \\
\hline$\lambda_{3}$ & 125,1 \\
\hline$\lambda_{4}$ & 213,4 \\
\hline
\end{tabular}




\begin{tabular}{|l|l|}
\hline$\lambda_{5}$ & 323,4 \\
\hline$\lambda_{6}$ & 442,2 \\
\hline
\end{tabular}

The following pictures Fig. $1-2$ show the graphs of eigenfunctions corresponding to the prescribed eigenvalues.

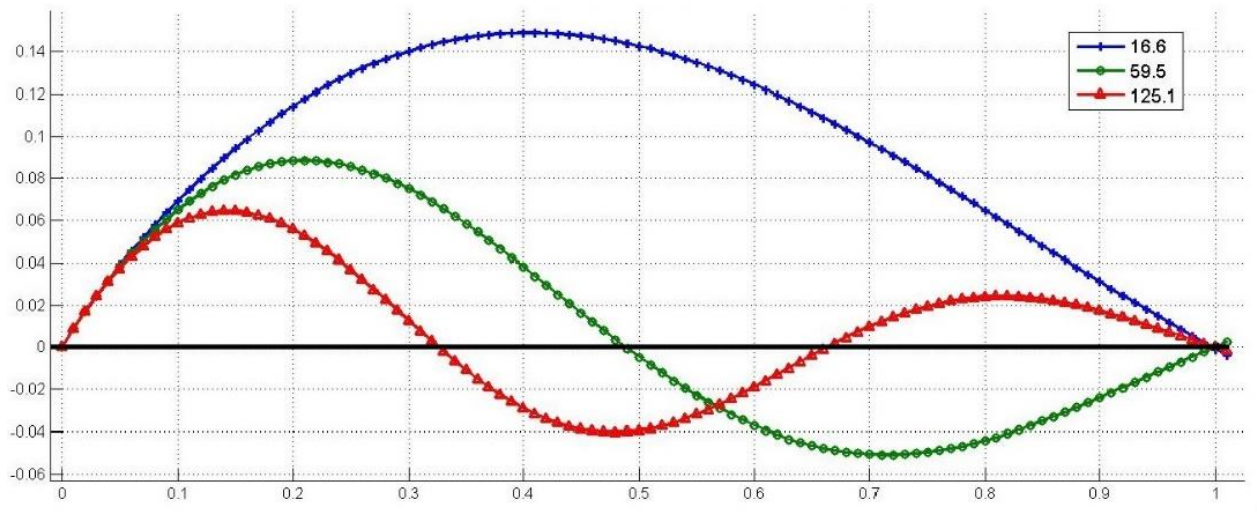

Fig. 1. Graphs of eigenfunctions $u_{1}(x), u_{2}(x), u_{3}(x)$ for $\lambda_{1}-\lambda_{3}$ (the first three) eigenvalues of the model.

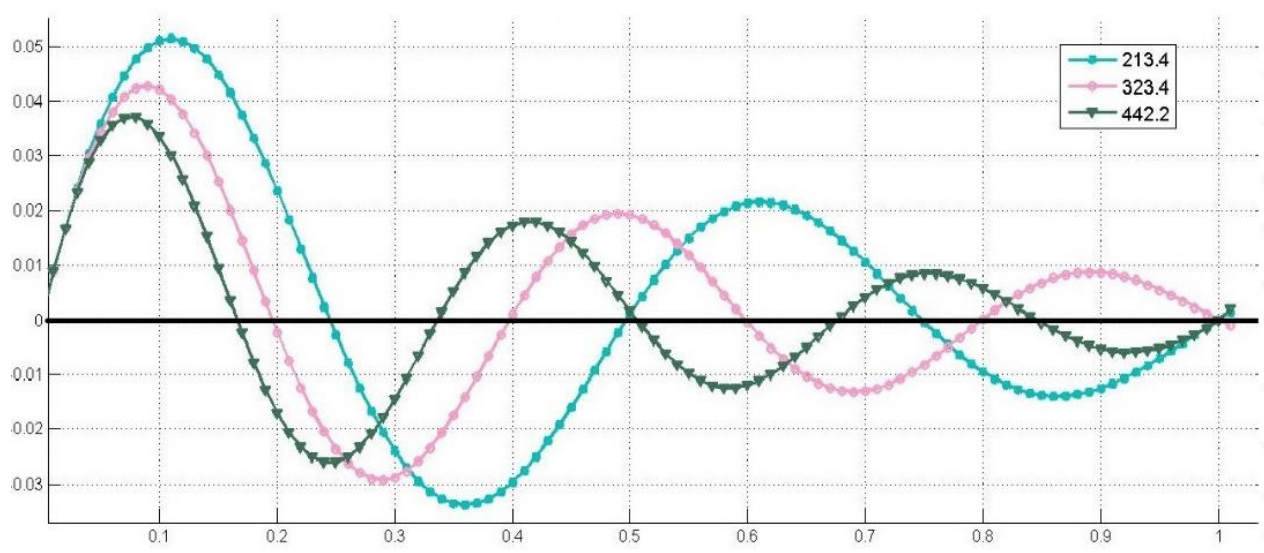

Fig. 2. Graphs of eigenfunctions $u_{4}(x), u_{5}(x), u_{6}(x)$ for $\lambda_{4}-\lambda_{6}$ (the second three) eigenvalues of the model.

\section{Conclusions}

The presence of the following basic oscillation properties follows from analysis of Fig. 1 2:

1) all eigenvalues are real and positive;

2) eigenvibration with the lowest frequency has no nodes;

3 ) eigenvibration with the frequency $\lambda_{k}$ ( $k$-th obertone) has exactly $k$ nodes;

$4)$ the nodes of two consecutive obertones are resurveyed. 


\section{References}

1. R. L. Bagley, P. J. Torvik. J. Rheolog., 27, 3 (1983)

2. R. L. Bagley, P. J. Torvik. AIAA Journal, 21, 5 (1983)

3. A. A. Chirkii, I. I. Matichin. Trudy instituta matematiki i mekhaniki UrO RAN. 1, 2 (2011)

4. D. Ingman, J. Suzdalnitsky. Comput. Methods Appl. Mech. Engrg. 193 (2004).

5. D. Ingman, J. Suzdalnitsky. Comput. Methods Appl. Mech. Engrg. 190 (2001)

6. G. K. Koh, J. Kelly. Earthquake engineering and structural dynamics. 19 (1990)

7. Gh. E. Draganescu, N. Cofan, D. L. Rujan. J. Optoelectron. Adv. Mater. 7 , 2 (2005)

8. A. Fenlander. AIAA J. 34, 5 (1996).

9. R. P. Meilanov, M. S. Pis'ma v ZhTF, 28, 1 (2002).

10. S. G. Samko, A. A. Kilbas, O. I. Marichev. Integraly i proizvodnye drobnogo poriadka i nekotorye ikh prilozheniia. (Minsk, Nauka i tekhnika, 1987)

11. T. S. Aleroev, S. V. Erokhin. Matem. Mod., 30, 7 (2018)

12. B. Ibrahim, Q. Dong, Z. Fan. Journal of nonlinear sciences and applications, 10 (2017)

13. E. R. Kekharsaeva, V. G. Pirozhkov. Mekhanika kompozitsionnykh materialov $i$ konstruktsii, slozhnykh i geterogennykh sred. (Moskva, IPRIM RAN, 2016).

14. Aleroev T.S., Kirianova L.V. Special Issueon Fractional Dynamical Systems and Applications (to be published) 